\title{
NMR protocol for determination of oxidation susceptibility of serum lipids and application of the protocol to a chocolate study
}

\author{
Tuulia Tynkkynen · Jaakko Mursu • Tarja Nurmi • \\ Kari Tuppurainen • Reino Laatikainen • \\ Pasi Soininen
}

Received: 17 March 2011/Accepted: 20 May 2011/Published online: 3 June 2011

(C) The Author(s) 2011. This article is published with open access at Springerlink.com

\begin{abstract}
A protocol for determination of oxidation susceptibility of serum lipids based on proton nuclear magnetic resonance $\left({ }^{1} \mathrm{H}\right.$ NMR) spectroscopy is presented and compared to the commonly used spectrophotometric method. Even though there are methodological differences between these two methods, the NMR-based oxidation susceptibility correlates well $\left(r^{2}=0.73\right)$ with the lag time determined spectrophotometrically. In addition to the oxidizability of serum lipids, the NMR method provides also information about the lipid profile. The NMR oxidation assay was applied to the chocolate study including fasting serum samples $(n=45)$ from subjects who had consumed white (WC), dark (DC) or high-polyphenol chocolate (HPC) daily for 3 weeks. The oxidation susceptibility of serum lipids decreased in the HPC group, and there was a significant difference between the WC and HPC groups $(P=0.031)$. According to the random forest analysis, the consumption of the HPC chocolate induced changes to the
\end{abstract}

Electronic supplementary material The online version of this article (doi:10.1007/s11306-011-0323-2) contains supplementary material, which is available to authorized users.

T. Tynkkynen $(\bowtie) \cdot$ K. Tuppurainen $\cdot$ R. Laatikainen · P. Soininen

Department of Biosciences, University of Eastern Finland,

Kuopio, Finland

e-mail: tuulia.tynkkynen@uef.fi

J. Mursu

School of Public Health, University of Minnesota,

Minneapolis, MN, USA

J. Mursu - T. Nurmi

Department of Public Health, Institute of Public Health and Clinical Nutrition, University of Eastern Finland Kuopio, Finland amounts of HDL, phosphatidylcholine, sphingomyelin, and nervonic, docosahexaenoic and myristic acids. Furthermore, arachidonic, docosahexaenoic, docosapentaenoic and palmitic acids, gamma-glutamyl transferase, hemoglobin, HDL, phosphatidylcholine and choline containing phospholipids explained about $60 \%$ of the oxidation susceptibility values.

Keywords Oxidation susceptibility $\cdot{ }^{1} \mathrm{H}$ NMR spectroscopy $\cdot$ Copper induced oxidation $\cdot$ Serum . Chocolate $\cdot$ Random forest

\section{Introduction}

Oxidative stress is commonly defined as a state in which the levels of reactive oxygen and nitrogen species, which oxidize lipids, proteins and nucleic acids in cells, and the relevant neutralizing substances are imbalanced (Opara 2006). The interest for studying the oxidative stress is mainly due to its probable role in many pathological conditions, for example in coronary artery disease and cancer (Delimaris et al. 2007; Serdar et al. 2006).

Human serum contains a variety of lipids of which the polyunsaturated fatty acids (PUFAs) in cholesterol esters, phospholipids, and triglycerides are subject to free radicalinitiated oxidation (Niki 2009; Schneider 2009; Uchida 2003). The free radical-mediated lipid peroxidation proceeds by chain mechanism yielding conjugated dienic lipid hydroperoxides as primary reaction products (Niki 2009; Pierini and Punta 2006). These hydroperoxides can be reduced to corresponding hydroxy fatty acids or decomposed either into various aldehydes or, if the original fatty acid is arachidonic acid, into isoprostanes (Dotan et al. 2004; Mlakar and Spiteller 1996). 
The methods to assess oxidative stress can be divided into three categories (Dotan et al. 2004). Firstly, there are assays that measure the concentrations of antioxidants (Mastaloudis et al. 2001; Sies and Krinsky 1995; Finckh et al. 1995; Dhariwal et al. 1990) or oxidation products of lipids (Roberts and Morrow 2000; Esterbauer et al. 1989), proteins (Fukagawa et al. 2001; Buss et al. 1997) and DNA (Shen and Ong 2000; Halliwell 2000). These methods require the measurements of several different compounds, which may exist in very low concentrations, be unstable or partly unknown. Secondly, there are various assays that assess the reductive capacity of body fluids, as well as the capacity of these fluids to inhibit oxidation (Re et al. 1999; Winston et al. 1998; Benzie and Strain 1996; Wayner et al. 1985). However, various antioxidants react differently in each assay, and thus, the interpretation of the results is not straightforward (Young 2001). Thirdly, there are assays that evaluate the oxidation susceptibility of lipids from serum or separated lipoproteins by exposing the sample under oxidative conditions (Delimaris et al. 2008; Smith et al. 2002; Schnitzer et al. 1995). The separation of lipoproteins is time consuming, and also some loss of watersoluble antioxidants may occur during the separation procedure. The methods measuring the oxidation susceptibility of whole serum are faster, and they also take the effects of serum antioxidants into account.

Oxidation susceptibility of lipids from whole serum is usually determined by initiating the oxidation with copper, which leads to the formation of conjugated dienic hydroperoxides that can be monitored spectrophotometrically since they absorb in the UV range (Delimaris et al. 2007; Schnitzer et al. 1998; Spranger et al. 1998). The result is expressed either as the lag time preceding oxidation, which describes the time before the maximal reaction rate of diene accumulation has been reached, or as the maximal rate of accumulation of absorbing products (Delimaris et al. 2008). However, the serum must be considerably diluted (up to 150-fold) to enable the spectrophotometric measurements of lipid oxidation products. Furthermore, also albumin and the other compounds of serum absorb in the UV range, thus, disturbing the detection of conjugated dienes (Schnitzer et al. 1995, 1998).

The objective of this work was to develop a method to study the oxidation susceptibility of serum lipids by proton nuclear magnetic resonance $\left({ }^{1} \mathrm{H} \mathrm{NMR}\right)$ spectroscopy. This measurement can be conveniently combined with the NMR measurement of the serum lipid profile (Tukiainen et al. 2008). Previously, NMR spectroscopy has been applied to study lipid peroxidation of low density lipoproteins (LDL) (Soininen et al. 2007; Corso et al. 1997; Lodge et al. 1995; Lodge et al. 1993), and the behavior of the signal arising from the bisallylic protons has shown to be a good measure for the peroxidation state of LDL particles (Lodge et al.
1995). However, NMR has not been used to study the oxidation susceptibility of lipids from whole serum.

The developed NMR oxidation assay was applied to serum samples from the study subjects who had consumed white (WC), dark (DC) or high-polyphenol (HPC) chocolate (Mursu et al. 2004) daily for 3 weeks, and the results were compared with the lag time values obtained using the spectrophotometric method. Random forest (RF) approach was used to find out the metabolic changes induced by the chocolate consumption and to assess the determinants for the oxidation susceptibility of serum lipids.

\section{Materials and methods}

\subsection{Subjects and study setting}

The oxidation method was developed using serum obtained from three healthy volunteers. The blood samples were drawn a few days before the first experiments, and the serum was stored at $-70^{\circ} \mathrm{C}$. The developed oxidation protocol was applied to the chocolate study including fasting serum samples from the subjects who had consumed $75 \mathrm{~g}$ of WC, DC or HPC chocolate daily for 3 weeks (Mursu et al. 2004). The blood samples were taken before and after the chocolate consumption. The serum samples of the chocolate study were stored at $-70^{\circ} \mathrm{C}$ and not thawed before. The study population consisted of 45 nonsmoking volunteer men $(n=12)$ and women $(n=33)$ that fulfilled all the following criteria: (i) BMI $<32 \mathrm{~kg} / \mathrm{m}^{2}$; (ii) no regular use of any drugs or supplements with antioxidative ( $\beta$-carotene, vitamins $\mathrm{C}$ or $\mathrm{E}$ ) or lipid-lowering properties; (iii) no chronic diseases such as diabetes, coronary heart disease, or other major illnesses; and (iv) willingness to consume $75 \mathrm{~g}$ of study chocolate daily for 3 weeks. The study protocol was approved by the Research Ethics Committee, Hospital District of Northern Savo, Finland.

\subsection{Sample preparation}

The samples to determine the oxidation susceptibility of serum lipids were prepared as follows. First, $0.3 \mathrm{ml}$ of serum, $0.3 \mathrm{ml}$ of NMR buffer and $0.3 \mathrm{ml}$ of $0.15 \mathrm{M}$ sodium chloride solution were combined in a $2 \mathrm{ml}$ eppendorf tube, and the solution was warmed in an incubator at $37^{\circ} \mathrm{C}$ for $10 \mathrm{~min}$. In the sample preparation, the same volumes of serum and NMR buffer were used as for the serum NMR metabonomics measurements (Soininen et al. 2009) so that these two protocols can be combined in future. Oxidation was initiated by adding $0.1 \mathrm{ml}$ of $5,10,20$ or $40 \mathrm{mM}$ $\mathrm{CuSO}_{4}$ to the prewarmed solution to yield a copper concentration of $0.5,1.0,2.0$ or $4.0 \mathrm{mM}$, respectively. 
To determine the initial concentration of PUFAs in each sample, also a reference sample without copper was prepared. The copper containing samples were incubated in an end-over-end mixer at $37^{\circ} \mathrm{C}$ for $1.5,3,4.5$ or $6 \mathrm{~h}$.

The oxidation reaction was terminated and the lipids were extracted by using a modified Folch method (Folch et al. 1957). Briefly, $10 \mathrm{ml}$ of $1: 2(\mathrm{v} / \mathrm{v})$ methanol/dichloromethane solution and $10 \mathrm{ml}$ of $0.15 \mathrm{M}$ sodium chloride solution were added to the incubated sample. The sample was mixed in a vertical shaker for $5 \mathrm{~min}$ and centrifuged (Hettich Rotina 46R, $2400 \times g, 10 \mathrm{~min}$, at $+4^{\circ} \mathrm{C}$ ). The lower dichloromethane phase was recovered and the aqueous phase was extracted again with $5 \mathrm{ml}$ of dichloromethane. The separated organic phases were combined and evaporated to dryness. The lipids were redissolved into $0.6 \mathrm{ml}$ of $\mathrm{CDCl}_{3}$ containing $0.03 \%$ tetramethylsilane for chemical shift reference.

To evaluate the repeatability of the method, five replicate samples were prepared within period of 2 months in random order. The chocolate study samples were analyzed in triplicates (WC and HPC groups) or duplicates (DC group).

\subsection{NMR spectroscopy}

${ }^{1} \mathrm{H}$ NMR spectra were recorded on a Bruker AVANCE 500 DRX (Bruker, Karlsruhe, Germany) spectrometer operating at $500.13 \mathrm{MHz}$ equipped with a $5 \mathrm{~mm}$ QNP probe. Shimming and tuning of the samples were performed manually. The spectra were measured at $22^{\circ} \mathrm{C}$, and a standard Bruker proton pulse sequence $(\mathrm{zg})$ with a $90^{\circ}$ pulse was employed. The number of scans was 64, the number of dummy scans 2 , the spectral width $4.4 \mathrm{kHz}$, and the number of data points $64 \mathrm{~K}$. The total acquisition time was $7.45 \mathrm{~s}$ with the delay time (d1) of $0.5 \mathrm{~s}$.

The spectra were processed using PERCH NMR Software (Laatikainen et al. 2011). The measured free induction decays were multiplied by an exponential window function $(\mathrm{LB}=0.5 \mathrm{~Hz})$ to increase the signal-to-noise ratio.

\subsection{Quantification of lipids and calculation of the NMR oxidation susceptibility}

The areas of the lipid resonances were determined using lineshape fitting analysis (Tukiainen et al. 2008). The structures of some multiplets (for example, see Fig. 1), which were defined by the coupling constants, were used as constraints that enabled the quantitative analysis of severely overlapping peaks (Soininen et al. 2005). The signal areas of the bisallylic protons from PUFAs (A $A_{\text {PUFA }}$ ) at 2.74-2.88 ppm were used to determine the NMR oxidation susceptibility. First, the difference between the amounts of PUFAs before (REF) and after $(\mathrm{OX})$ the oxidation reaction was calculated. Then the difference was divided by the signal area of PUFAs from the spectrum of non-oxidized sample and converted to a percentage value $\left(\left(A_{\text {PUFA }}(R E F)-A_{\text {PUFA }}\right.\right.$ $\left.(\mathrm{OX})) / \mathrm{A}_{\mathrm{PUFA}}(\mathrm{REF}) \times 100 \%\right)$. The obtained value describes the amount of oxidized PUFAs, and thus, the oxidation susceptibility of serum lipids. The signal areas were scaled so that the signal area of total cholesterol $\mathrm{C}(18) \mathrm{H}_{3}$ protons was the same both in the spectra of non-oxidized and oxidized serum. It should be noted that many of the cholesterol oxidation products can be identified from ${ }^{1} \mathrm{H}$ NMR spectrum since their $\mathrm{C}(18) \mathrm{H}_{3}$ signals resonate at $0.61-0.69 \mathrm{ppm}$ (Bradamante et al. 1992), next to the non-oxidized cholesterol $\mathrm{C}(18) \mathrm{H}_{3}$ signals (0.676 and $\left.0.678 \mathrm{ppm}\right)$. Thus, it is important to take all these signal areas into account when calculating the total cholesterol amount that is used as a reference in the NMR protocol.

\subsection{Statistics}

The results are expressed as means ( \pm standard deviations). Changes between the baseline and end-point values within the study groups were tested by paired $t$-test. Means were compared across the study groups by the analysis of variance (ANOVA), and post hoc Tukey's test was used when a statistically significant heterogeneity between the groups was shown by the ANOVA. Correlations were estimated by Pearson's or Spearman's correlation coefficients. Differences with $P$ values of 0.05 or less were considered significant. SPSS software (version 14.0; SPSS, Inc., Chicago, IL, USA) was used for the statistical analyses.

Random forest approach was used to study the chocolate induced metabolic effects as well as to explore the descriptors for the oxidation susceptibility. This method, introduced by Breiman (Breiman 2001) and not common at the time of the original work (Mursu et al. 2004), allowed us to gain more insights into the previously obtained results. Random forests are ensembles of unpruned decision trees. For classification, the trees are combined using majority voting, one vote for each tree. For regression, predictions are generated by averaging over individual trees. In general, the performance of random forests compares favorably with modern machine learning methods such as support vector machines or neural networks (Hastie et al. 2008). Among multivariate methods, random forests are particularly resistant to overfitting, including only two adjustable parameters; the number of trees (Ntree) and the number of variables to be tried in each split (Mtry). Ntree is typically set at 500-2000, as a larger number will provide no extra gain. For Mtry, a value of sqrt(Nvar) is recommended for classification and Nvar/3 for regression; here Nvar is the number of variables. The optimization of Mtry usually provides a slight improvement only. Distinctively, random forests come with a build-in cross-validation using out-of-bag data (usually $30 \%$ of the 


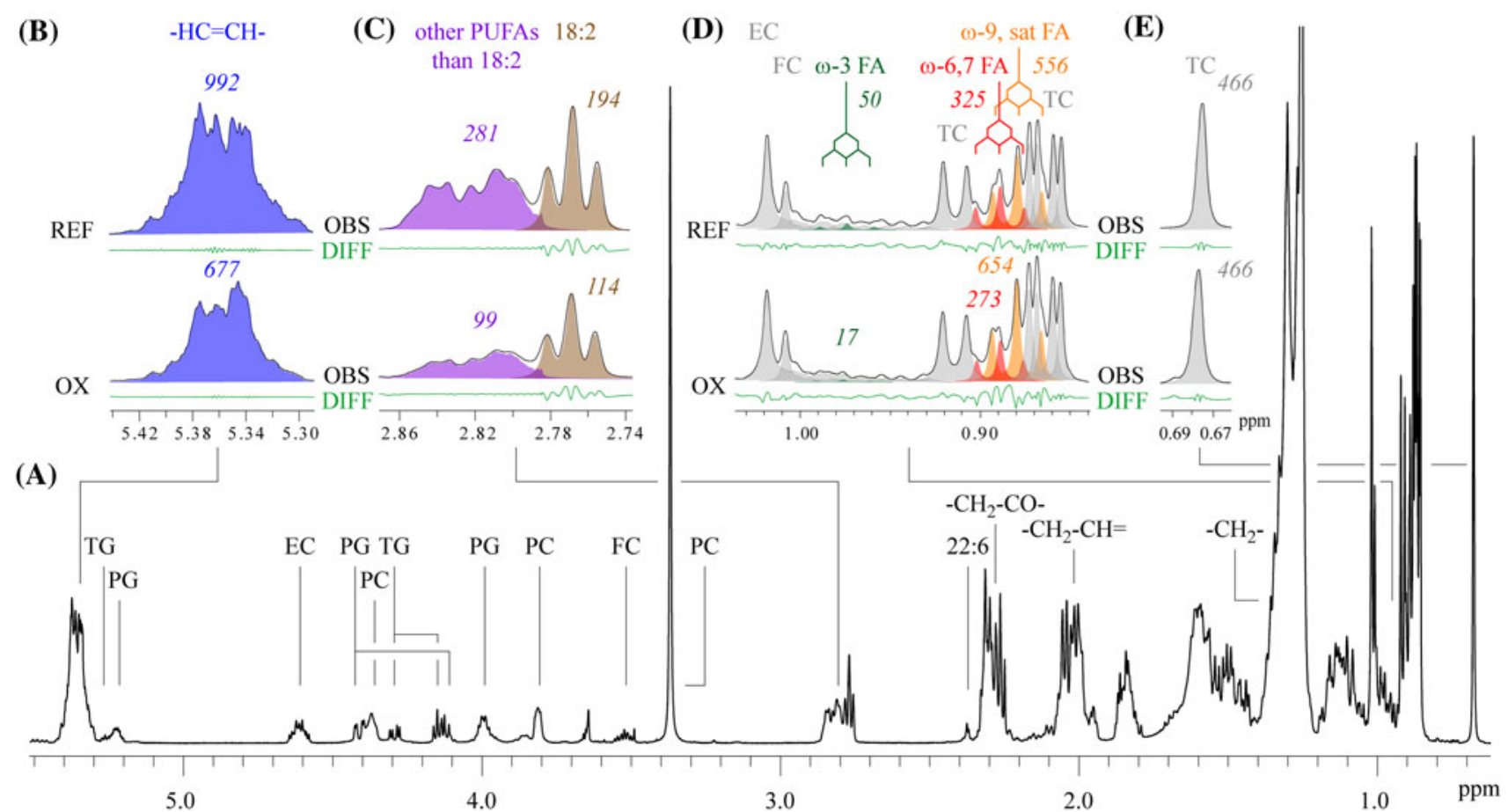

Fig. 1 A ${ }^{1} \mathrm{H}$ NMR spectrum of extracted serum with signal assignments (a). Some essential parts of the spectra (b-e) before and after the oxidation are shown at the top of the figure. The signals arising from the double bond protons (b) and the bisallylic protons from PUFAs (c) decrease during oxidation. There are also changes in the amounts of different fatty acids (d). Cholesterol oxidizes slightly

samples). Herewith an estimate for the prediction error is achieved as a by-product, and so there is no actual need for cross-validation with separate train and test sets. There are also two heuristic ways of measuring variable importance in classification, mean decrease in classification accuracy and mean decrease in Gini index. These standard performance indices usually provide slightly different results, and there is no consensus opinion which one should be preferred. The reliability of the prediction can simply be estimated by counting the votes for each class-a sharp voting profile, favoring strongly a certain class, is usually a hallmark of reliability. For regression, the corresponding performance measures are increase in mean square error and increase in node purity. The calculations were performed with the $\mathrm{R}$ (R Development Core Team 2008) program package randomForest (Liaw and Wiener 2002).

\section{Results and discussion}

\subsection{Development and optimization of the NMR oxidation assay}

The oxidation of PUFAs can be seen from a ${ }^{1} \mathrm{H}$ NMR spectrum of extracted serum by following the signal arising under the conditions used and these oxysterol $\mathrm{C}(18) \mathrm{H}_{3}$ signals resonate at $0.61-0.69 \mathrm{ppm}$. The signal areas are referenced to total cholesterol $\mathrm{C}(18) \mathrm{H}_{3}$ signals including also the oxidized forms (e). $E C$ esterified cholesterol, $F A$ fatty acid, $F C$ free cholesterol, $P C$ phosphatidylcholine, $P G$ phosphoglyceride, sat saturated, $T C$ total cholesterol, $T G$ triglyceride

from the bisallylic protons at $2.74-2.88 \mathrm{ppm}$ that decreases when the oxidation reaction proceeds (Fig. 1). In addition, the signals from the double bond protons $(5.30-5.45 \mathrm{ppm})$ as well as the $\omega-3(0.98 \mathrm{ppm})$ and $\omega-6$ and $\omega-7$ fatty acid methyl proton signals $(0.89 \mathrm{ppm})$ decrease during oxidation whereas the $\omega-9$ and saturated fatty acid signal (0.88 ppm) increases (Fig. 1).

Several copper concentrations $(0.5,1.0,2.0$ and $4.0 \mathrm{mM})$ as well as incubation times (1.5, 3, 4.5 and $6 \mathrm{~h})$ were tested in order to find out the copper concentration and the time point that yield maximal amount of information about the oxidation susceptibility of serum lipids as well as the largest variance between individuals. As can be seen from Fig. 2a, the oxidation reaction proceeds rapidly when copper concentrations of 1-4 $\mathrm{mM}$ are used. The copper concentration of $0.5 \mathrm{mM}$ provides milder oxidation conditions and the oxidation proceeds slower, which also results in larger variance between individuals. The Fig. $2 b$ shows that the largest variance between the percentage values describing the amount of oxidized PUFAs of the three subjects is obtained using incubation time of 6 hours. In addition, at this point, the speed of the oxidation reaction has slowed down allowing slight differences in the incubation times without affecting the results. 

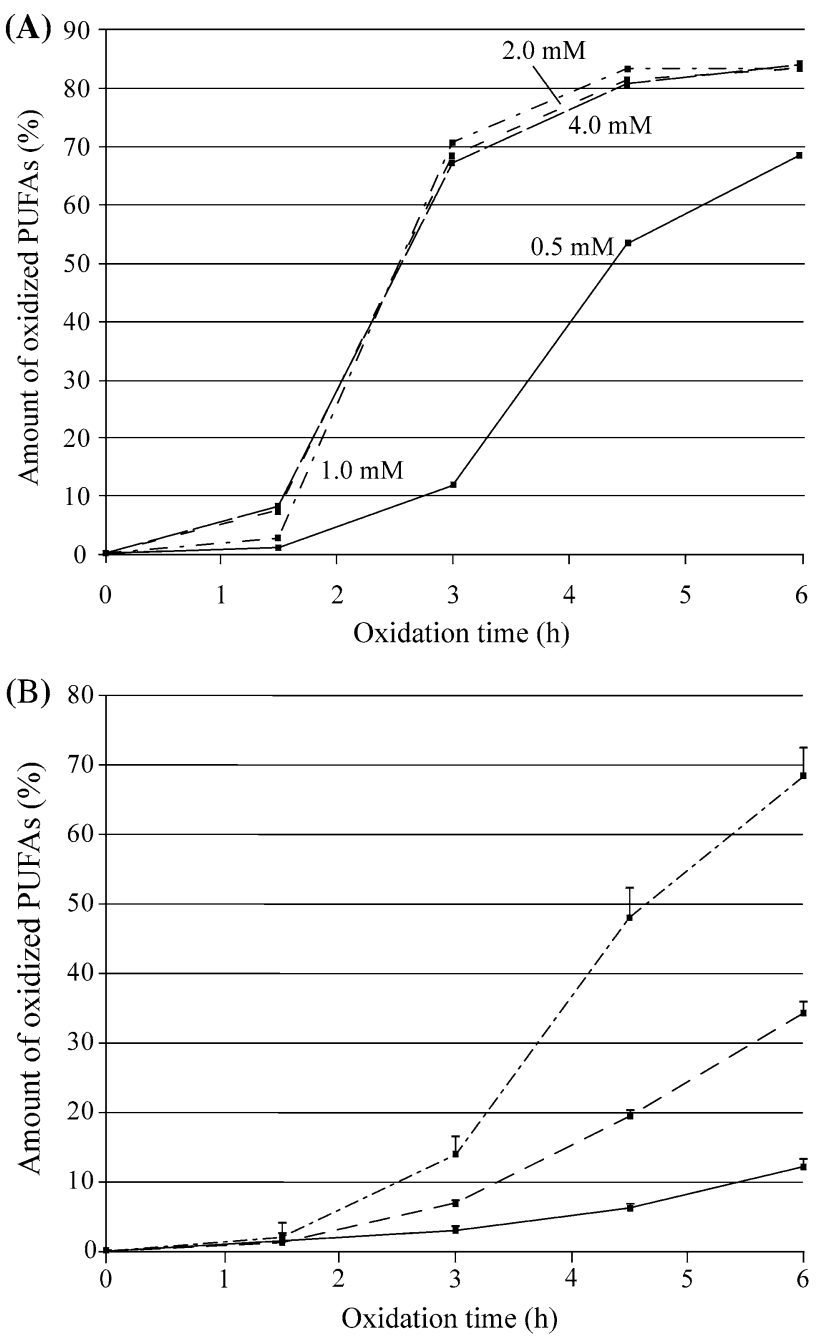

Fig. 2 The amounts of oxidized PUFAs during the copper induced oxidation with four different copper concentrations (a) and with a copper concentration of $0.5 \mathrm{mM}$ for three subjects (b). Each data point in $\mathbf{b}$ is a mean of five determinations measured within period of 2 months

The repeatability of the assay was studied by oxidizing five replicate serum samples from three volunteers within period of 2 months in random order (Fig. 2b). At the time points 1.5 and $3 \mathrm{~h}$, the deviations in the oxidation susceptibility range between 0.4 and 2.8 percentage units. At the later time points $(4.5$ and $6 \mathrm{~h})$, the standard deviations are within 0.5 and 4.3 percentage units.

\subsection{Application of the NMR oxidation assay to chocolate study samples}

The optimized oxidation assay $\left(0.5 \mathrm{mM} \mathrm{CuSO}_{4}\right.$ and $6 \mathrm{~h}$ incubation) was applied to fasting serum samples from the subjects who had consumed $75 \mathrm{~g}$ of WC, DC or HPC chocolate for 3 weeks. For the samples, several factors, including the oxidation susceptibility of serum lipids determined spectrophotometrically, were determined earlier (Mursu et al. 2004).

The amounts of oxidized PUFAs before and after the chocolate consumption for each of the study subject are illustrated in Electronic Supplementary Fig. S1. The NMR oxidation assay showed that the oxidation susceptibility of serum lipids decreased in the HPC group $(P=0.016)$ after the chocolate consumption but there were no significant changes in the DC $(P=0.407)$ or WC $(P=0.758)$ groups. The change in the NMR oxidation susceptibility was significantly different between the WC and HPC groups $(P=0.031)$. As can be seen from Table 1 and Electronic Supplementary Fig. S1, the baseline values of the HPC group are lower than in the $\mathrm{WC}$ and DC groups. Thus, the analyses were also performed by adjusting the baseline values of the variables. However, the changes between the groups remained significantly different even after the adjustment $(P=0.043)$.

\subsection{Correlation between the NMR oxidation assay and the spectrophotometric method}

The baseline and end-point oxidation susceptibility values determined by ${ }^{1} \mathrm{H}$ NMR were plotted against the corresponding lag time values, and the strong correlation between these two methods (Spearman's correlation coefficient $-0.825, P<0.001$ ) is illustrated in Fig. 3. If a sample is very susceptible to oxidation, the lag time is short. By using the NMR method, this is seen as a high amount of oxidized PUFAs. However, even though the baseline and end-point values of the spectrophotometric and NMR method correlate, the change values (end-baseline), which are used in the ANOVA analysis, do not correlate (Pearson's correlation coefficient -0.158 , $P=0.317$ ), and only the NMR oxidation assay reveals significant differences between the chocolate groups.

The different results obtained by the NMR and spectrophotometric assays can be, at least partly, explained by the different settings of these experiments. Firstly, the spectrophotometric method measures the time before the maximal reaction rate of diene accumulation has been reached, and the NMR method gives the amount of oxidized PUFAs after 6-h oxidation. Thus, the NMR method observes the overall oxidation reaction considering both the early and later stages of the reaction whereas the spectrophotometric assay focuses on the early stages of the oxidation reaction. Secondly, there are different incubation temperatures $\left(37^{\circ} \mathrm{C}\right.$ for the NMR protocol and $30^{\circ} \mathrm{C}$ for the spectrophotometric method), which have been shown to affect to the kinetics of LDL oxidation (Ramos et al. 1995). Also different copper-to-serum ratios $(0.5 \mathrm{mM} / 300 \mu \mathrm{l}$ for the NMR method and $0.05 \mathrm{mM} / 13.4 \mu \mathrm{l}$ for the spectrophotometric assay) may have some effect on the oxidation 
Table 1 The NMR oxidation susceptibility (\%) and the lag time (min) before (baseline) and after (change) the consumption of study chocolates for 3 weeks

\begin{tabular}{|c|c|c|c|c|c|c|c|}
\hline \multirow[t]{2}{*}{ Parameter } & \multicolumn{2}{|l|}{ WC } & \multicolumn{2}{|l|}{ DC } & \multicolumn{2}{|l|}{ HPC } & \multirow[t]{2}{*}{$P$} \\
\hline & Baseline & Change & Baseline & Change & Baseline & Change & \\
\hline Susceptibility & $72 \pm 14$ & $0 \pm 5^{\mathrm{b}}$ & $71 \pm 10$ & $-2 \pm 8^{\mathrm{b}}$ & $64 \pm 17$ & $-7 \pm 10^{\mathrm{b}}$ & 0.033 \\
\hline Lag time ${ }^{\mathrm{a}}$ & $118 \pm 43$ & $6 \pm 31^{\mathrm{c}}$ & $122 \pm 35$ & $4 \pm 32^{c}$ & $160 \pm 61$ & $-3 \pm 28^{\mathrm{c}}$ & 0.711 \\
\hline
\end{tabular}

Data expressed as means $\pm \mathrm{SD} ; P$ for the differences in changes between the groups (one-way ANOVA); $W C$ white chocolate, $D C$ dark chocolate, $H P C$ high-polyphenol chocolate

${ }^{a}$ The values of this assay were determined previously (Mursu et al. 2004)

b $n=15$

${ }^{\mathrm{c}} n=14$

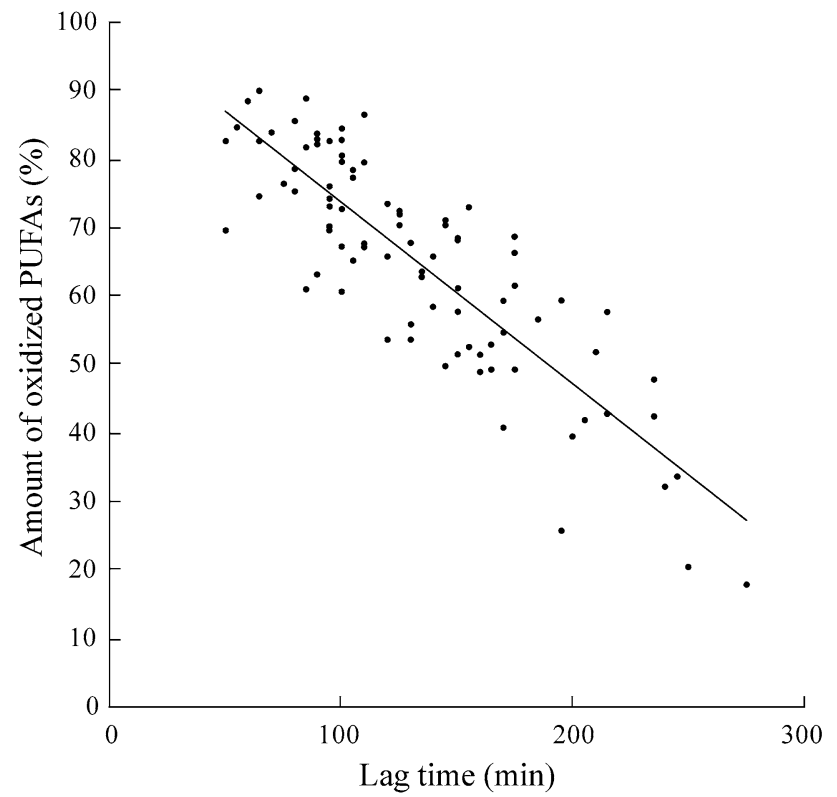

Fig. 3 The amounts of oxidized PUFAs after the copper induced oxidation determined by the ${ }^{1} \mathrm{H}$ NMR method plotted against the corresponding lag time values of the oxidation monitored spectrophotometrically from the chocolate study samples. The regression line is $y=-0.2665 x+100.4, r^{2}=0.7308$

reactions. Thirdly, the NMR method is more specific since there are no disturbing signals in the spectrum overlapping with the signals arising from PUFAs whereas in the spectrophotometric assay also albumin and cholesterol oxidation products, mainly 7-ketocholesterol, absorb at the same wavelength as conjugated dienes (Schnitzer et al. 1998).

\subsection{Variables used for the random forest analyses}

Random forest method was used to study the chocolate consumption induced metabolic changes as well as to assess the determinants for the oxidation susceptibility of serum lipids. The variables included into the analyses consisted of previously measured variables (Mursu et al. 2004) and a total of 23 lipid variables obtained by NMR, which are presented in Table 2. Some of the variables were determined using two different methods. For example, the concentrations of triglycerides, linoleic acid and docosahexaenoic acid measured using the NMR method and an enzymatic colorimetric test or gas chromatography were comparable; the correlations between the concentrations determined with the two different methods were very strong (correlation coefficients 0.964, 0.966 and 0.959, respectively, $P<0.001$ for all).

\subsection{Metabolic profiling}

The chocolates administered had similar amounts and profiles of fatty acids, excluding behenic acid, but differed in the amounts of cocoa and polyphenols (mainly catechin, epicatechin and procyanidins). The cocoa content of the DC and HPC chocolates was rather similar but the HPC chocolate contained $557 \mathrm{mg}$ polyphenols in $100 \mathrm{~g}$ chocolate whereas the corresponding amount in the DC chocolate was $366 \mathrm{mg}$.

The RF method allowed clustering of the WC, DC and HPC groups with six variables (Fig. 4), and the out-of-bag error was $22 \%$. Into the analysis, the change (end-baseline) values of the variables shown in Table 2, except behenic acid, were included. Since the HPC chocolate contained tenfold amount of behenic acid $(0.35 \mathrm{~g} / 100 \mathrm{~g}$ chocolate $)$ compared with the WC $(0.048 \mathrm{~g} / 100 \mathrm{~g}$ chocolate $)$ and DC $(0.041 \mathrm{~g} / 100 \mathrm{~g}$ chocolate) chocolates, the increase in behenic acid concentration after the consumption of the HPC chocolate is not a consequence of endogenous metabolic effects. The inclusion of behenic acid into the analysis would elevate it to the most important variable causing the clustering (Electronic Supplementary Fig. S2), but, at the same time, it would prevent the discovery of the other metabolically more relevant variables.

The variable profiles for each chocolate group are shown in Fig. 5, and it appears that the HPC group has different variable profiles than the WC and DC groups. These results suggest that the consumption of the HPC chocolate induces 
Table 2 The variables included into the RF analyses

\begin{tabular}{|c|c|}
\hline Number & Variable \\
\hline 1 & Hemoglobin (g/l) \\
\hline 2 & Hematocrit \\
\hline 3 & Erythrocytes $\left(\times 10^{12} / 1\right)$ \\
\hline 4 & Leucocytes $\left(\times 10^{9} / 1\right)$ \\
\hline 5 & $\operatorname{MCV}(\mathrm{fl})$ \\
\hline 6 & Thrombocytes $\left(\times 10^{9} / 1\right)$ \\
\hline 7 & Total serum cholesterol $(\mathrm{mmol} / \mathrm{l})^{\mathrm{a}}$ \\
\hline 8 & LDL-cholesterol $(\mathrm{mmol} / \mathrm{l})^{\mathrm{b}}$ \\
\hline 9 & HDL-cholesterol $(\mathrm{mmol} / \mathrm{l})^{\mathrm{c}}$ \\
\hline 10 & Serum TGs $(\mathrm{mmol} / \mathrm{l})^{\mathrm{a}}$ \\
\hline 11 & Creatinine $(\mu \mathrm{mol} / \mathrm{l})^{\mathrm{d}}$ \\
\hline 12 & $\operatorname{ASAT}(\mathrm{U} / \mathrm{l})^{\mathrm{d}}$ \\
\hline 13 & $\operatorname{ALAT}(\mathrm{U} / \mathrm{l})^{\mathrm{d}}$ \\
\hline 14 & B-glucose (mmol/l) \\
\hline 15 & $\gamma-\mathrm{GT}(\mathrm{U} / \mathrm{l})^{\mathrm{d}}$ \\
\hline 16 & Myristic acid, 14:0 $(\%)^{\mathrm{e}}$ \\
\hline 17 & Palmitic acid, 16:0 $(\%)^{\mathrm{e}}$ \\
\hline 18 & Palmitoleic acid, 16:1 $(\%)^{\mathrm{e}}$ \\
\hline 19 & Stearic acid, 18:0 $(\%)^{\mathrm{e}}$ \\
\hline 20 & Oleic acid, $18: 1(\%)^{\mathrm{e}}$ \\
\hline 21 & Linoleic acid, 18:2 $(\%)^{\mathrm{e}}$ \\
\hline 22 & $\alpha$-linolenic acid, $18: 3 \omega-3(\%)^{\mathrm{e}}$ \\
\hline 23 & 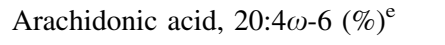 \\
\hline 24 & Eicosatetraenoic acid, 20:4 $1 \omega-3(\%)^{\mathrm{e}}$ \\
\hline 25 & Eicosapentaenoic acid, 20:5 (\%) \\
\hline 26 & $\gamma$-linolenic acid, $18: 3 \omega-6(\%)^{\mathrm{e}}$ \\
\hline 27 & Docosahexaenoic acid, 22:6 (\%) \\
\hline 28 & Docosapentaenoic acid, 22:5 $(\%)^{\mathrm{e}}$ \\
\hline 29 & Behenic acid, 22:0 $(\%)^{\mathrm{e}}$ \\
\hline 30 & Lignoceric acid, $24: 0(\%)^{\mathrm{e}}$ \\
\hline 31 & Nervonic acid, 24:1 $(\%)^{\mathrm{e}}$ \\
\hline 32 & Retinol $(\mu \mathrm{g})^{\mathrm{f}}$ \\
\hline 33 & Carotene $(\mu \mathrm{g})^{\mathrm{f}}$ \\
\hline 34 & Vitamin $D(\mu \mathrm{g})^{\mathrm{f}}$ \\
\hline 35 & Vitamin $E(m g)^{\mathrm{f}}$ \\
\hline 36 & Thiamine $\mathrm{B}_{1}(\mathrm{mg})^{\mathrm{f}}$ \\
\hline 37 & Riboflavin $B_{2}(m g)^{f}$ \\
\hline 38 & Niacin $B_{3}(m g)^{f}$ \\
\hline 39 & Pyridoxine $\mathrm{B}_{6}(\mathrm{mg})^{\mathrm{f}}$ \\
\hline 40 & Cobalamin $B_{12}(\mu g)^{f}$ \\
\hline 41 & Folate $B_{9}(\mu \mathrm{g})^{\mathrm{f}}$ \\
\hline 42 & Pantothenic acid $B_{5}(m g)^{f}$ \\
\hline 43 & Biotin $B_{7}(\mu g)^{f}$ \\
\hline 44 & Vitamin $C(m g)^{f}$ \\
\hline 45 & Calcium $(m g)^{\mathrm{f}}$ \\
\hline 46 & Magnesium (mg) ${ }^{\mathrm{f}}$ \\
\hline 47 & $\operatorname{Iron}(\mathrm{mg})^{\mathrm{f}}$ \\
\hline 48 & Selenium $(\mu \mathrm{g})^{\mathrm{f}}$ \\
\hline
\end{tabular}

Table 2 continued

\begin{tabular}{|c|c|}
\hline Number & Variable \\
\hline 49 & Mercury $(\mu \mathrm{g})^{\mathrm{f}}$ \\
\hline 50 & Flavonoids $(\mathrm{mg})^{\mathrm{f}}$ \\
\hline 51 & Quercetin $(\mathrm{mg})^{\mathrm{f}}$ \\
\hline 52 & Myricetin $(m g)^{f}$ \\
\hline 53 & Kaempferol $(\mathrm{mg})^{\mathrm{f}}$ \\
\hline 54 & Alcohol $(\mathrm{E} \%)^{\mathrm{f}}$ \\
\hline 55 & Total serum cholesterol $(\mathrm{mmol} / \mathrm{l})$ \\
\hline 56 & Esterified cholesterol (mmol/l) \\
\hline 57 & Free cholesterol $(\mathrm{mmol} / \mathrm{l})$ \\
\hline 58 & Total FAs (mmol/l) \\
\hline 59 & $18: 2(\%)$ \\
\hline 60 & Other PUFAs than 18:2 (mmol/l) \\
\hline 61 & All PUFAs (mmol/l) \\
\hline 62 & $22: 6(\%)$ \\
\hline 63 & Total TG (mmol/l) \\
\hline 64 & Total PG (mmol/l) \\
\hline 65 & Phosphatidylcholine (mmol/l) \\
\hline 66 & Sphingomyelin (mmol/l) \\
\hline 67 & Total choline phospholipids (mmol/l) \\
\hline 68 & $\omega-3$ FAs $(\%)$ \\
\hline 69 & $\omega-6$ and $\omega-7$ FAs $(\%)$ \\
\hline 70 & $\omega-9$ and saturated FAs $(\%)$ \\
\hline 71 & Ratio of $\mathrm{CH}_{2}$ signal area to $\mathrm{FA}$ signal area \\
\hline 72 & Ratio of TG to PG \\
\hline 73 & Ratio of $\mathrm{CH}_{2}$ signal area to DB signal area \\
\hline 74 & Ratio of DB signal area to FA signal area \\
\hline 75 & Ratio of PUFAs to DB signal area \\
\hline 76 & Ratio of PUFAs to FA \\
\hline 77 & Average FA chain length \\
\hline
\end{tabular}

The variables 1-54 were determined previously (Mursu et al. 2004) whereas the variables $55-77$ were obtained from ${ }^{1} \mathrm{H}$ NMR spectrum and were scaled so that the total serum cholesterol values determined both in the previous study (variable 7) and by the ${ }^{1} \mathrm{H}$ NMR method (variable 55) are identical. The baseline and end-point values $\left(\mathrm{VAR}_{\mathrm{BASE}+\mathrm{END}}\right)$ or the change $\left(\mathrm{VAR}_{\mathrm{CHANGE}}\right)$ values of these variables were used depending on the RF analysis

$A L A T$ alanine transaminase, $A S A T$ aspartate transaminase, $D B$ double bond, $F A$ fatty acid, $M C V$ mean cell volume, $P G$ phosphoglyceride, $P U F A$ polyunsaturated fatty acid, $T G$ triglyceride, $\gamma-G T$, gammaglutamyl transferase

${ }^{a}$ Determined using an enzymatic colorimetric test

${ }^{b}$ Determined by a direct cholesterol measurement (Konelab)

c Measured from the supernatant after magnesium chloride dextran sulphate precipitation

${ }^{d}$ Measured using a Clinical Chemistry Analyzer (Konelab)

e Serum fatty acids were extracted using chloroform-methanol and methylated with sulphuric acid-methanol. The methylated fatty acids were analyzed by a gas chromatograph

${ }^{\mathrm{f}}$ Based on the 4 day food record that was collected before the intervention and during the last week of the supplementation period 

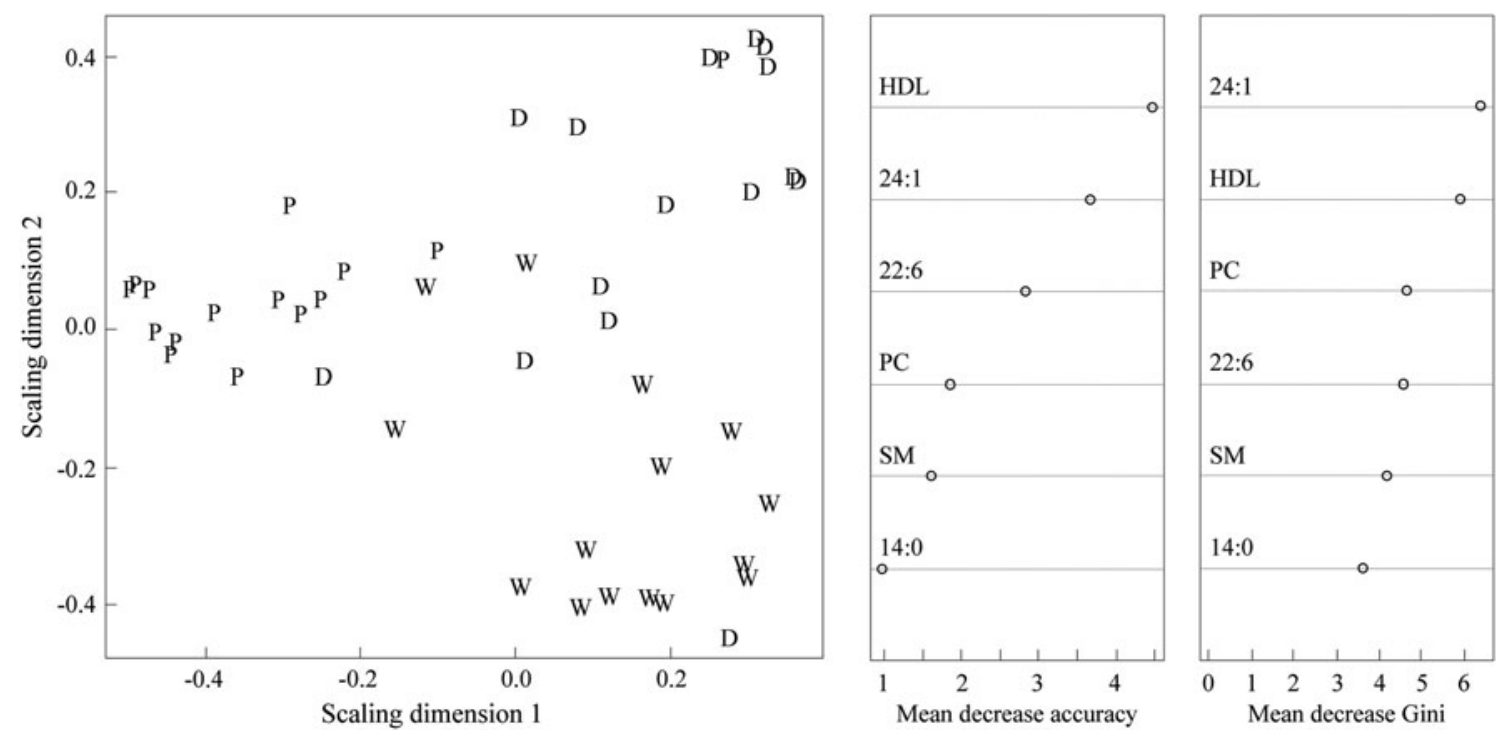

Fig. 4 RF clustering of the chocolate study samples [WC $(W)$, DC $(D)$ and HPC $(P)$ groups] with the change variables shown in Table 2 excluding behenic acid. The clustering was obtained with six variables and the variable importance measures, mean decrease in

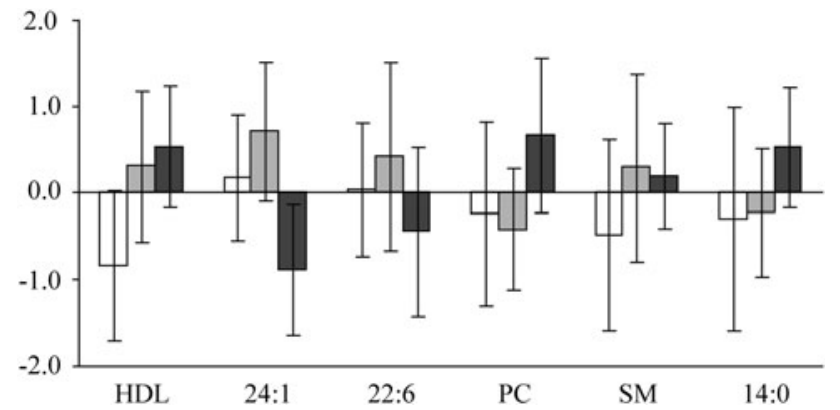

Fig. 5 The profiles of the variable changes for the WC (white), DC (light grey), and HPC (dark grey) groups. The values are expressed as standard deviation units from the mean of the whole study population. 24:1 nervonic acid, 22:6 docosahexaenoic acid, $P C$ phosphatidylcholine, $S M$ sphingomyelin, 14:0 myristic acid

different changes to the amounts of HDL, nervonic acid, docosahexaenoic acid, phosphatidylcholine, sphingomyelin and myristic acid than the consumption of the WC or DC chocolates. Since the main difference between the study chocolates was the amount of polyphenols, it is likely that they have induced the metabolic changes in the HPC group but on the grounds of this study it is impossible to identify the mechanisms of action.

Previously, the effects of cocoa and chocolate have been studied in cell-cultures, in laboratory animals and in humans, and the results are reviewed by Rimbach and coworkers (Rimbach et al. 2009). There have been a variety of study settings, and also the outcomes are diverse. The effects obtained from human interventions include, e.g., decrease in LDL oxidation (Mathur et al. 2002), decrease accuracy and mean decrease in Gini index, are shown for each of these variables. 24:1 nervonic acid; 22:6 docosahexaenoic acid, $P C$ phosphatidylcholine, $S M$ sphingomyelin, 14:0 myristic acid

of blood pressure (Faridi et al. 2008), increased HDL concentration (Baba et al. 2007), improved endothelial function (Schroeter et al. 2006), and increased insulin sensitivity (Grassi et al. 2005). Also, there are studies with rats which have shown that flavonoids can act by modulating fatty acid metabolism (Dihal et al. 2008; de Boer et al. 2006; Ohta et al. 2006). For example, quercetin, which is also present in cocoa (Sanbongi et al. 1998), upregulates genes involved in fatty acid catabolism and lowers the fatty acid levels in plasma (de Boer et al. 2006).

\subsection{Determinants of oxidation susceptibility of serum lipids}

Random forest approach was also used to assess the determinants for the oxidation susceptibility of serum lipids for the chocolate study samples. The analysis included the baseline and end-point values of the variables (Table 2). The correlation coefficients between the baseline and end-point variables $\left(\mathrm{VAR}_{\mathrm{BASE}+\mathrm{END}}\right)$ and the corresponding NMR oxidation susceptibility values (NMROX $\mathrm{BASE}_{\mathrm{END}}$ ) as well as between the variables $\left(\mathrm{VAR}_{\mathrm{BASE}+\mathrm{END}}\right)$ and the lag time values $\left(\mathrm{LAG}_{\mathrm{BASE}+\mathrm{END}}\right)$ are shown in Fig. 6 . Due to the inverse correlation of the values of the NMR oxidation method and the spectrophotometric assay, the signs of the correlation coefficients are opposite for these two methods. To ease the comparison, the signs of the coefficients of the spectrophotometric method were inversed. As can be seen from Fig. 6, the correlation coefficients for the NMR oxidation method and the spectrophotometric assay are very similar and most of the correlations are relatively weak (less 


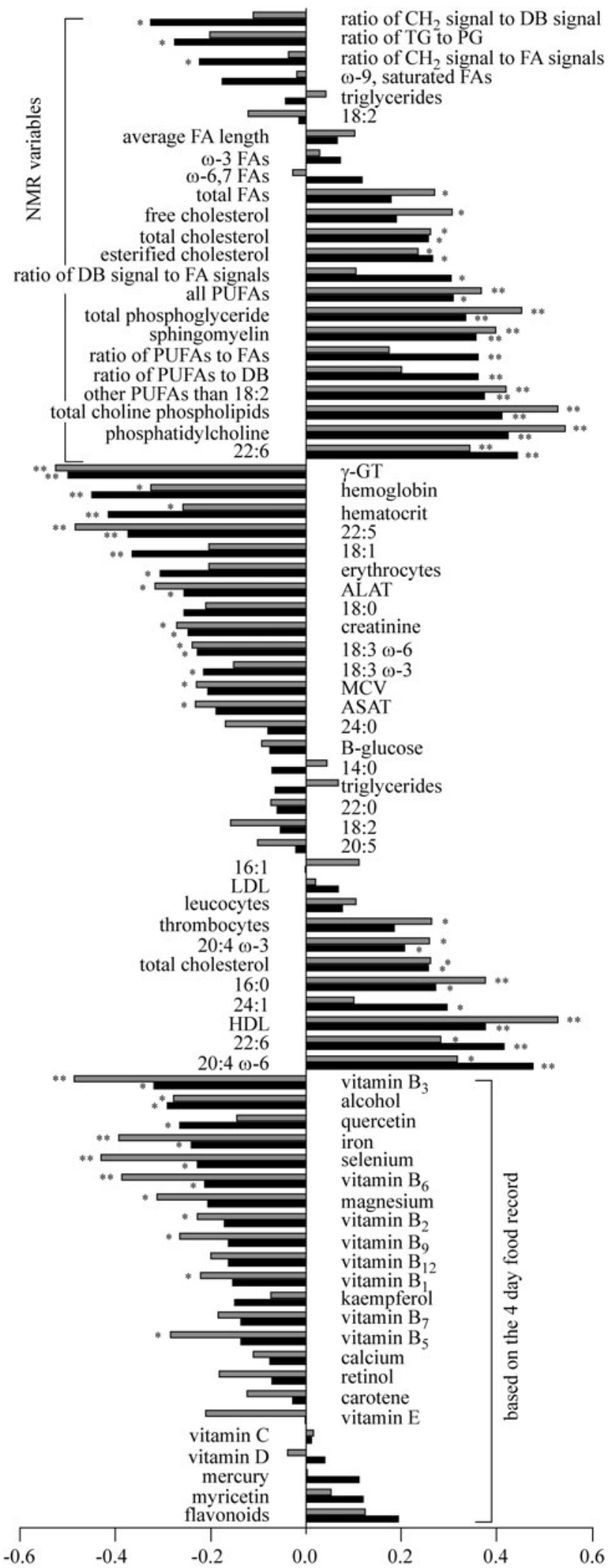

than $10.4 \mid$ ). The $\mathrm{NMROX}_{\mathrm{BASE}+\mathrm{END}}$ values have the strongest correlations with gamma-glutamyl transferase $(\gamma-\mathrm{GT})$, arachidonic acid and hemoglobin (Spearman's correlation
4 Fig. 6 The correlations between the variables $\left(\mathrm{VAR}_{\mathrm{BASE}+\mathrm{END}}\right)$ (shown in Table 2) and the NMR oxidation susceptibility (NMROX BASE+END) values (black) and the lag time ( $\left.\mathrm{LAG}_{\mathrm{BASE}+\mathrm{END}}\right)$ values (grey). Since the NMR oxidation susceptibility values and the lag time values are inversely correlated, the inverse correlation coefficients for the spectrophotometric method are presented in order to ease the comparison. $P$-values are indicated with one $(0.001<P \leq 0.05)$ or two $(P \leq 0.001)$ asterisks

coefficients $-0.498,0.477$ and -0.449 , respectively, $P<0.001$ for all) whereas the $\mathrm{LAG}_{\mathrm{BASE}+\mathrm{END}}$ values correlate strongest with phosphatidylcholine, HDL and $\gamma$-GT (Spearman's correlation coefficients $0.544,0.528$ and -0.525 , respectively, $P<0.001$ for all). For example, the positive correlation coefficient of arachidonic acid means that the NMR oxidation susceptibility increases when the amount of arachidonic acid increases. For the spectrophotometric method, the high amount of arachidonic acid corresponds to a short lag time value.

The purpose of the RF analysis was to find out if the oxidation susceptibilities could be explained with the determined variables (Table 2). However, the RF analysis was not straightforward. As can be expected from the relatively weak correlations (Fig. 6), the performance of the regression model is weak and the picture of the mutual importance of the variables remains somewhat unclear since it may change from case to case considerably. The reason for this behavior is that the differences in importance are usually very small for most of the variables. This, together with the small number of samples (only 15 for each chocolate group), tends to make the RF regression procedure unstable. In these conditions the proper variable selection is not a trivial task.

In order to cope with this problem, the following strategy was applied. First, we derived a large number of RF models $(n=200)$ for both the NMROX BASE+END $_{\text {and }}$ $\mathrm{LAG}_{\mathrm{BASE}+\mathrm{END}}$ values, and then the importance values were scaled to the maximum and the data sets were combined. After that an average importance was calculated for each variable (Fig. 7), and the variables with the highest average importance values were selected for the RF models trying to explain the $\mathrm{NMROX}_{\mathrm{BASE}+\mathrm{END}}$ and $\mathrm{LAG}_{\mathrm{BASE}+\mathrm{END}}$ values.

$\mathrm{RF}$ regression was able to explain $60 \%$ of the NMROX $X_{\text {BASE+END }}$ values and $62 \%$ of the $\mathrm{LAG}_{\mathrm{BASE}+\mathrm{END}}$ values with nine variables (Fig. 8). Most of these descriptors are lipid variables including arachidonic acid, docosahexaenoic acid, docosapentaenoic acid, phosphatidylcholine, HDL, choline containing phospholipids and palmitic acid. Previously, the decreased oxidation susceptibility has been related to low amounts of PUFAs (Eritsland 2000; Bonanome et al. 1992), and also here, arachidonic and docosahexaenoic acids are positively correlated with the oxidation 


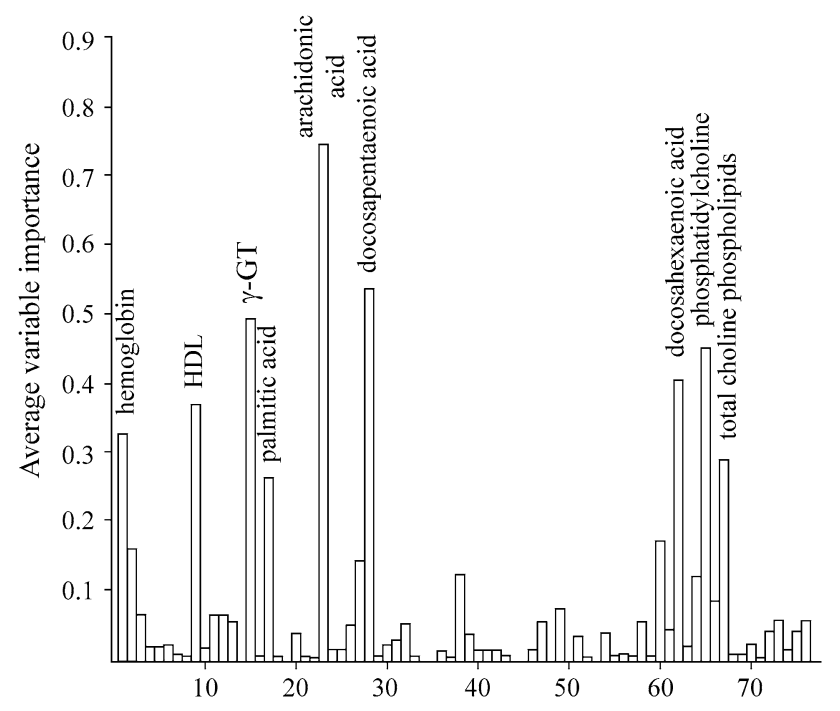

Fig. 7 Average variable importances for the variables shown in Table 2. The numbering on the $x$-axis follows the numbering of the variables in Table 2

susceptibility (Fig. 6). The amount of docosapentaenoic acid acts differently but it is a less abundant fatty acid in serum [approximately $0.2 \mathrm{~mol} \%$ from all serum fatty acids (Hodson et al. 2008)], and thus, has less effect on overall oxidation susceptibility than arachidonic and docosahexaenoic acids. As can be seen from Fig. 6, the increased oxidation susceptibility is related to increased amounts of phosphatidylcholine and total choline containing phospholipids. This high oxidizability of these phospholipids is probably due to the fact that they contain PUFAs at their $s n-2$ position of the glycerol backbone. Also the correlation of high HDL concentrations with high oxidation susceptibility (Fig. 6) is in line with the previous findings (Shimonov et al. 1999; Nyyssönen et al. 1997). HDL particles contain high amounts of lipid hydroperoxides (Nyyssönen et al. 1997), and thus, shorten the lag time and increase the amount of oxidized PUFAs.

In addition to the lipid variables, also hemoglobin and serum $\gamma$-GT are descriptors of the NMROX BASE+END $_{\text {and }}$ $\mathrm{LAG}_{\mathrm{BASE}+\mathrm{END}}$ values, and they are inversely correlated with the oxidation susceptibility (Fig. 6). It has been shown that hemoglobin can catalyze various oxidative reactions (Alayash et al. 2001). Since hemoglobin containing red blood cells have been removed from the serum samples, the possible oxidative reactions have been occurred prior to the blood separation. Elevated serum $\gamma$-GT activity has usually been used as a marker for excessive alcohol consumption but it has also been suggested a marker for oxidative stress (Lee et al. 2004). However, our findings propose opposite. There is evidence of the antioxidative role of cellular $\gamma$-GT but the relationship between serum $\gamma$ GT and cellular $\gamma$-GT is not known (Lee et al. 2004). Serum $\gamma$-GT may also be an indirect marker of some other mechanism related to oxidation susceptibility.
Fig. 8 Increase in mean square error (IncMSE) (\%) and increase in node purity (Inc node purity) values for each variable obtained from the RF regression when modeling the oxidation susceptibility values (NMROX $\mathrm{BASE+END}$ explained with $\mathrm{VAR}_{\mathrm{BASE}+\mathrm{END}}$ ) and the lag time values $\left(\mathrm{LAG}_{\mathrm{BASE}+\mathrm{END}}\right.$ explained with $\left.\mathrm{VAR}_{\mathrm{BASE}+\mathrm{END}}\right)$. 16:0 palmitic acid, $20: 4 \omega-6$ arachidonic acid, 22:5 docosapentaenoic acid, 22:6 docosahexaenoic acid, $H B$ hemoglobin, $P C$ phosphatidylcholine, tot chol total choline phospholipids

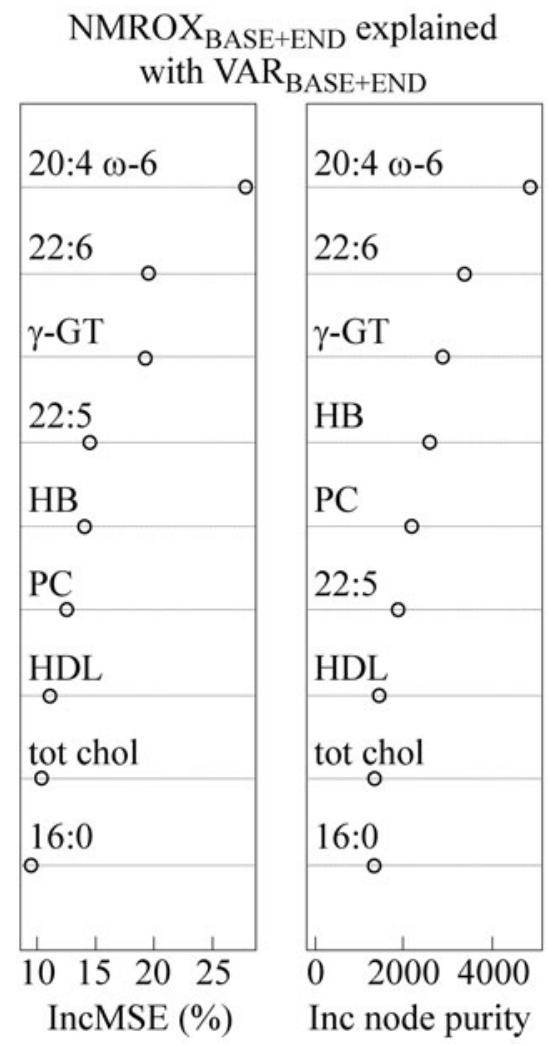

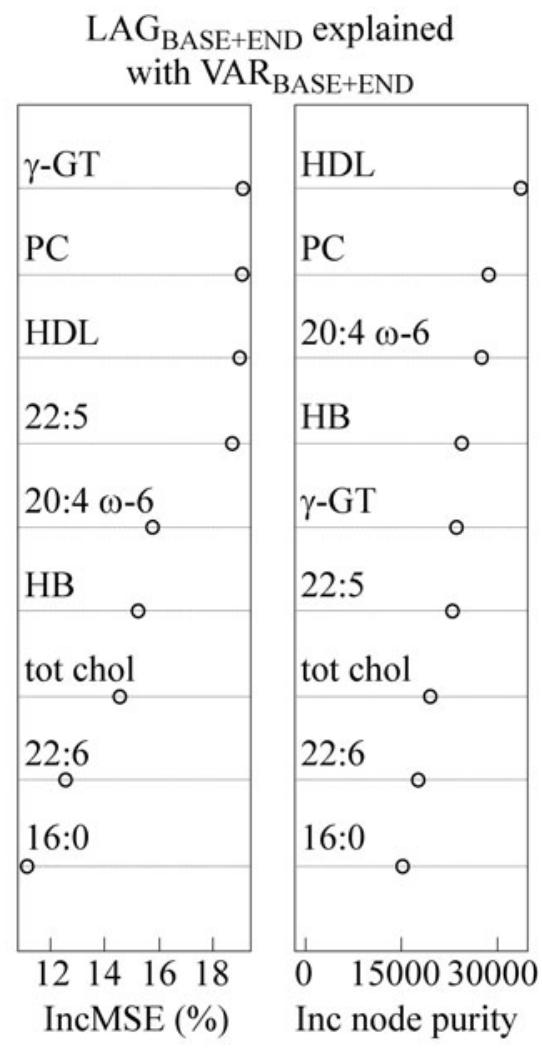


The variance importances for the descriptors of the $\mathrm{RF}$ regression models for the NMROX $\mathrm{BASE}_{\mathrm{END}}$ and $\mathrm{LAG}_{\mathrm{BASE}+\mathrm{END}}$ values are shown in Fig. 8. Arachidonic acid and docosahexaenoic acid seem to be more important determinants for the NMROX $\mathrm{BASE}_{\mathrm{END}}$ values than for the $\mathrm{LAG}_{\mathrm{BASE}+\mathrm{END}}$ values, whereas phosphatidylcholine and HDL have higher variable importance values in the model explaining $\mathrm{LAG}_{\mathrm{BASE}+\mathrm{END}}$ values. Since the NMR oxidation protocol measures the amount of oxidized PUFAs, it is logical that the PUFA variables are highlighted among the descriptors.

Previously, the determinants of serum lipid oxidation resistance have been studied by multivariate-regression analysis (Nyyssönen et al. 1997). In that study, vitamin C and urate were proposed to be the strongest determinants of plasma antioxidant capacity (Nyyssönen et al. 1997). Also, $\alpha$-tocopherol, albumin and HDL concentration have been shown to have a strong impact on serum lipid resistance to oxidation (Nyyssönen et al. 1997). Among our RF results, only HDL cholesterol of these was recognized as a determinant of oxidation susceptibility. However, considering that in our data serum urate and albumin concentrations were lacking and that the vitamin $\mathrm{C}$ and $\alpha$-tocopherol values were based on a 4 day food record, the explanation percentages of the models trying to explain the NMROX $_{\text {BASE+END }}$ and $\mathrm{LAG}_{\mathrm{BASE}+\mathrm{END}}$ values (60 and $62 \%$, respectively) are quite high. We also suggest that both the NMR oxidation and spectrophotometric methods yield invaluable measures that contain information on the metabolic state that is related to oxidation susceptibility, which can vary greatly between individuals.

\section{Concluding remarks}

In this study, a repeatable NMR-based protocol for studying the oxidation susceptibility of serum lipids was developed and assessed. In addition to the oxidizability of serum lipids, the NMR protocol yields vast amount of metabolic descriptors, if combined with the NMR metabonomic analysis of serum. The NMR protocol was applied to the serum samples of the chocolate study, and the oxidation susceptibility decreased significantly in the HPC group. This could not be detected by using the spectrophotometric method in the original study (Mursu et al. 2004), even though the values obtained by these two oxidation methods correlated $\left(r^{2}=0.73\right)$. The random forest analysis revealed that the consumption of the HPC chocolate induced changes to the amounts of HDL, phosphatidylcholine, sphingomyelin, and nervonic, docosahexaenoic and myristic acids. Furthermore, the RF analysis was able to explain about $60 \%$ of the oxidation susceptibility values with nine descriptors, including arachidonic, docosahexaenoic, docosapentaenoic and palmitic acids, gamma-glutamyl transferase, hemoglobin, HDL, phosphatidylcholine and choline containing phospholipids. Since the administered chocolates had similar amounts and profiles of fatty acids, except behenic acid, the polyphenols could have altered the metabolism of the study subjects and induced the changes to the oxidation susceptibilities.

Acknowledgments This work was supported by the National Graduate School of Organic Chemistry and Chemical Biology (TT) and the Academy of Finland project number \#114526 (TN) and \#137870 (PS).

Open Access This article is distributed under the terms of the Creative Commons Attribution Noncommercial License which permits any noncommercial use, distribution, and reproduction in any medium, provided the original author(s) and source are credited.

\section{References}

Alayash, A. I., Patel, R. P., \& Cashon, R. E. (2001). Redox reactions of hemoglobin and myoglobin: Biological and toxicological implications. Antioxidants \& Redox Signaling, 3, 313-327.

Baba, S., Osakabe, N., Kato, Y., et al. (2007). Continuous intake of polyphenolic compounds containing cocoa powder reduces LDL oxidative susceptibility and has beneficial effects on plasma HDL-cholesterol concentrations in humans. American Journal of Clinical Nutrition, 85, 709-717.

Benzie, I. F. F., \& Strain, J. J. (1996). The ferric reducing ability of plasma (FRAP) as a measure of "antioxidant power": The FRAP assay. Analytical Biochemistry, 239, 70-76.

Bonanome, A., Pagnan, A., Biffanti, S., et al. (1992). Effect of dietary monounsaturated and polyunsaturated fatty-acids on the susceptibility of plasma low-density lipoproteins to oxidative modification. Arteriosclerosis and Thrombosis, 12, 529-533.

Bradamante, S., Barenghi, L., Giudici, G. A., \& Vergani, C. (1992). Free radicals promote modifications in plasma high-density lipoprotein: nuclear magnetic resonance analysis. Free Radical Biology and Medicine, 12, 193-203.

Breiman, L. (2001). Random forests. Machine Learning, 45, 5-32.

Buss, H., Chan, T. P., Sluis, K. B., Domigan, N. M., \& Winterbourn, C. C. (1997). Protein carbonyl measurement by a sensitive ELISA method. Free Radical Biology and Medicine, 23, 361-366

Corso, G., Trivellone, E., Motta, A., et al. (1997). Effect of low density lipoprotein fatty acid composition on copper-induced peroxidation: ${ }^{1} \mathrm{H}$-nuclear magnetic resonance analysis. Clinica Chimica Acta, 258, 193-200.

de Boer, V. C. J., van Schothorst, E. M., Dihal, A. A., et al. (2006). Chronic quercetin exposure affects fatty acid catabolism in rat lung. Cellular and Molecular Life Sciences, 63, 2847-2858.

Delimaris, I., Faviou, E., Antonakos, G., Stathopoulou, E., Zachari, A., \& Dionyssiou-Asteriou, A. (2007). Oxidized LDL, serum oxidizability and serum lipid levels in patients with breast or ovarian cancer. Clinical Biochemistry, 40, 1129-1134.

Delimaris, I., Georgopoulos, S., Kroupis, C., et al. (2008). Serum oxidizability, total antioxidant status and albumin serum levels in patients with aneurysmal or arterial occlusive disease. Clinical Biochemistry, 41, 706-711. 
Dhariwal, K. R., Washko, P. W., \& Levine, M. (1990). Determination of dehydroascorbic acid using high-performance liquid chromatography with coulometric electrochemical detection. Analytical Biochemistry, 189, 18-23.

Dihal, A. A., van der Woude, H., Hendriksen, P. J. M., et al. (2008). Transcriptome and proteome profiling of colon mucosa from quercetin fed F344 rats point to tumor preventive mechanisms, increased mitochondrial fatty acid degradation and decreased glycolysis. Proteomics, 8, 45-61.

Dotan, Y., Lichtenberg, D., \& Pinchuk, I. (2004). Lipid peroxidation cannot be used as a universal criterion of oxidative stress. Progress in Lipid Research, 43, 200-227.

Eritsland, J. (2000). Safety considerations of polyunsaturated fatty acids. American Journal of Clinical Nutrition, 71, 197S-201S.

Esterbauer, H., Striegl, G., Puhl, H., \& Rotheneder, M. (1989). Continuous monitoring of invitro oxidation of human low-density lipoprotein. Free Radical Research Communications, 6, 67-75.

Faridi, Z., Njike, V. Y., Dutta, S., Ali, A., \& Katz, D. L. (2008). Acute dark chocolate and cocoa ingestion and endothelial function: A randomized controlled crossover trial. American Journal of Clinical Nutrition, 88, 58-63.

Finckh, B., Kontush, A., Commentz, J., Hübner, C., Burdelski, M., \& Kohlschütter, A. (1995). Monitoring of ubiquinol-10, ubiquinone-10, carotenoids, and tocopherols in neonatal plasma microsamples using high-performance liquid chromatography with coulometric electrochemical detection. Analytical Biochemistry, 232, 210-216.

Folch, J., Lees, M., \& Sloan Stanley, G. H. (1957). A simple method for the isolation and purification of total lipides from animal tissues. Journal of Biological Chemistry, 226, 497-509.

Fukagawa, N. K., Li, M., Timblin, C. R., \& Mossman, B. T. (2001). Modulation of cell injury and survival by high glucose and advancing age. Free Radical Biology and Medicine, 31, $1560-1569$.

Grassi, D., Lippi, C., Necozione, S., Desideri, G., \& Ferri, C. (2005). Short-term administration of dark chocolate is followed by a significant increase in insulin sensitivity and a decrease in blood pressure in healthy persons. American Journal of Clinical Nutrition, 81, 611-614.

Halliwell, B. (2000). Why and how should we measure oxidative DNA damage in nutritional studies? How far have we come? American Journal of Clinical Nutrition, 72, 1082-1087.

Hastie, T., Tibshirani, R., \& Friedman, J. (2008). The elements of statistical learning. New York: Springer.

Hodson, L., Skeaff, C. M., \& Fielding, B. A. (2008). Fatty acid composition of adipose tissue and blood in humans and its use as a biomarker of dietary intake. Progress in Lipid Research, 47, 348-380.

Laatikainen, R., Niemitz, M., Korhonen, S.-P., Hassinen, T., \& Venäläinen, T. (2011). PERCH NMR Software, http://www. perchsolutions.com.

Lee, D.-H., Blomhoff, R., \& Jacobs, D. R. (2004). Is serum gamma glutamyltransferase a marker of oxidative stress? Free Radical Research, 38, 535-539.

Liaw, A., \& Wiener, M. (2002). Classification and regression by randomForest. $R$ News, 2, 18-22.

Lodge, J. K., Patel, S. U., \& Sadler, P. J. (1993). Aldehydes from metal ion- and lipoxygenase-induced lipid peroxidation: detection by ${ }^{1}$ H-n.m.r. spectroscopy. Biochemical Journal, 289, $149-153$

Lodge, J. K., Sadler, P. J., Kus, M. L., \& Winyard, P. G. (1995). Copper-induced LDL peroxidation investigated by ${ }^{1} \mathrm{H}-\mathrm{NMR}$ spectroscopy. Biochimica et Biophysica Acta, 1256, 130-140.

Mastaloudis, A., Leonard, S. W., \& Traber, M. G. (2001). Oxidative stress in athletes during extreme endurance exercise. Free Radical Biology and Medicine, 31, 911-922.
Mathur, S., Devaraj, S., Grundy, S. M., \& Jialal, I. (2002). Cocoa products decrease low density lipoprotein oxidative susceptibility but do not affect biomarkers of inflammation in humans. Journal of Nutrition, 132, 3663-3667.

Mlakar, A., \& Spiteller, G. (1996). Previously unknown aldehydic lipid peroxidation compounds of arachidonic acid. Chemistry and Physics of Lipids, 79, 47-53.

Mursu, J., Voutilainen, S., Nurmi, T., et al. (2004). Dark chocolate consumption increases HDL cholesterol concentration and chocolate fatty acids may inhibit lipid peroxidation in healthy humans. Free Radical Biology and Medicine, 37, 1351-1359.

Niki, E. (2009). Lipid peroxidation: Physiological levels and dual biological effects. Free Radical Biology and Medicine, 47, 469-484.

Nyyssönen, K., Porkkala-Sarataho, E., Kaikkonen, J., \& Salonen, J. T. (1997). Ascorbate and urate are the strongest determinants of plasma antioxidative capacity and serum lipid resistance to oxidation in Finnish men. Atherosclerosis, 130, 223-233.

Ohta, Y., Sami, M., Kanda, T., Saito, K., Osada, K., \& Kato, H. (2006). Gene expression analysis of the anti-obesity effect by apple polyphenols in rats fed a high fat diet or a normal diet. Journal of Oleo Science, 55, 305-314.

Opara, E. C. (2006). Oxidative stress. Disease-a-Month, 52, 183-198.

Pierini, M., \& Punta, C. (2006). New developments in peroxidation of polyunsaturated fatty acids. Letters in Organic Chemistry, 3, 91-97.

R Development Core Team. (2008). R: A language and environment for statistical computing. Vienna: R Foundation for Statistical Computing.

Ramos, P., Gieseg, S. P., Schuster, B., \& Esterbauer, H. (1995). Effect of temperature and phase-transition on oxidation resistance of low-density-lipoprotein. Journal of Lipid Research, 36, 2113-2128.

Re, R., Pellegrini, N., Proteggente, A., Pannala, A., Yang, M., \& Rice-Evans, C. (1999). Antioxidant activity applying an improved ABTS radical cation decolorization assay. Free Radical Biology and Medicine, 26, 1231-1237.

Rimbach, G., Melchin, M., Moehring, J., \& Wagner, A. E. (2009). Polyphenols from cocoa and vascular health-A critical review. International Journal of Molecular Sciences, 10, 4290-4309.

Roberts, L. J., \& Morrow, J. D. (2000). Measurement of $\mathrm{F}_{2^{-}}$ isoprostanes as an index of oxidative stress in vivo. Free Radical Biology and Medicine, 28, 505-513.

Sanbongi, C., Osakabe, N., Natsume, M., Takizawa, T., Gomi, S., \& Osawa, T. (1998). Antioxidative polyphenols isolated from theobroma cacao. Journal of Agriculture and Food Chemistry, $46,454-457$.

Schneider, C. (2009). An update on products and mechanisms of lipid peroxidation. Molecular Nutrition \& Food Research, 53, 315-321.

Schnitzer, E., Pinchuk, I., Bor, A., Fainaru, M., Samuni, A. M., \& Lichtenberg, D. (1998). Lipid oxidation in unfractionated serum and plasma. Chemistry and Physics of Lipids, 92, 151-170.

Schnitzer, E., Pinchuk, I., Fainaru, M., Schafer, Z., \& Lichtenberg, D. (1995). Copper-induced lipid oxidation in unfractionated plasma: The lag preceding oxidation as a measure of oxidation-resistance. Biochemical and Biophysical Research Communications, 216, 854-861.

Schroeter, H., Heiss, C., Balzer, J., et al. (2006). (-)-Epicatechin mediates beneficial effects of flavanol-rich cocoa on vascular function in humans. Proceedings of the National Academy of Sciences of the United States of America, 103, 1024-1029.

Serdar, Z., Aslan, K., Dirican, M., Sarandöl, E., Yeşilbursa, D., \& Serdar, A. (2006). Lipid and protein oxidation and antioxidant status in patients with angiographically proven coronary artery disease. Clinical Biochemistry, 39, 794-803. 
Shen, H.-M., \& Ong, C.-N. (2000). Detection of oxidative DNA damage in human sperm and its association with sperm function and male infertility. Free Radical Biology and Medicine, 28, 529-536.

Shimonov, M., Pinchuk, I., Bor, A., et al. (1999). Susceptibility of serum lipids to copper-induced peroxidation correlates with the level of high density lipoprotein cholesterol. Lipids, 34, 255-259.

Sies, H., \& Krinsky, N. I. (1995). The present status of antioxidant vitamins and beta-carotene. American Journal of Clinical Nutrition, 62, S1299-S1300.

Smith, W. G., Reeves, C., Bibbs, D., \& Faas, F. H. (2002). Simple and rapid assessment of lipoprotein susceptibility to oxidation in the macromolecule fraction of plasma. Clinica Chimica Acta, 316, $19-24$.

Soininen, P., Haarala, J., Vepsäläinen, J., Niemitz, M., \& Laatikainen, R. (2005). Strategies for organic impurity quantification by ${ }^{1} \mathrm{H}$ NMR spectroscopy: Constrained total-line-shape fitting. Analytica Chimica Acta, 542, 178-185.

Soininen, P., Kangas, A. J., Würtz, P., et al. (2009). High-throughput serum NMR metabonomics for cost-effective holistic studies on systemic metabolism. Analyst, 134, 1781-1785.

Soininen, P., Öörni, K., Maaheimo, H., et al. (2007). ${ }^{1} \mathrm{H}$ NMR at $800 \mathrm{MHz}$ facilitates detailed phospholipid follow-up during atherogenic modifications in low density lipoproteins. Biochemical and Biophysical Research Communications, 360, 290-294.

Spranger, T., Finckh, B., Fingerhut, R., Kohlschütter, A., Beisiegel, U., \& Kontush, A. (1998). How different constituents of human plasma and low density lipoprotein determine plasma oxidizability by copper. Chemistry and Physics of Lipids, 91, 39-52.

Tukiainen, T., Tynkkynen, T., Mäkinen, V.-P., et al. (2008). A multimetabolite analysis of serum by ${ }^{1} \mathrm{H}$ NMR spectroscopy: Early systemic signs of Alzheimer's disease. Biochemical and Biophysical Research Communications, 375, 356-361.

Uchida, K. (2003). 4-Hydroxy-2-nonenal: A product and mediator of oxidative stress. Progress in Lipid Research, 42, 318-343.

Wayner, D. D. M., Burton, G. W., Ingold, K. U., \& Locke, S. (1985). Quantitative measurement of the total, peroxyl radical-trapping antioxidant capability of human blood plasma by controlled peroxidation: The important contribution made by plasma proteins. FEBS Letters, 187, 33-37.

Winston, G. W., Regoli, F., Dugas, A. J., Fong, J. H., \& Blanchard, K. A. (1998). A rapid gas chromatographic assay for determining oxyradical scavenging capacity of antioxidants and biological fluids. Free Radical Biology and Medicine, 24, 480-493.

Young, I. S. (2001). Measurement of total antioxidant capacity. Journal of Clinical Pathology, 54, 339. 